\title{
Femtosecond fiber-feedback OPO
}

\author{
T. Südmeyer, J. Aus der Au, R. Paschotta, and U. Keller \\ Ultrafi ast Laser Physics, Institute of Quarnen Electronics \\ Swiss Federal Instituse of Technology, ETH Hönggerberg - HPT, CH-8093 Zürich, Switzerland
}

\author{
P. G. R. Smith, G. W. Ross, and D. C. Hanna \\ Optoelectronics Research Censre, University of Southampton \\ Southampion SOI7 IBJ, United Kingdom
}

\begin{abstract}
We demonstrate the first synchronously pumped high-gain optical parametric oscillator with feedback through a single-mode fiber. We obtained $27 \mathrm{~W}$ in 900 -fs pulses at $1.45 \mu \mathrm{m}$. An active cavity length stabilization is not required.
\end{abstract}

\section{Introduction}

Synchronously pumped optical parametric oscillators (OPOs) are attractive sources of broadly wavelength-tunable ultrashor pulses as required for many applications. In this paper, we present a novel type of synchronously pumped $\mathrm{OPO}$, which is based on feedback through a single-mode fiber in combination with a very high gain in the nonlinear medium. Our concept lead to a very stable and compact OPO setup which is unusually insensitive against intracavity losses and drifts of the OPO cavity length. Even with non-optimized optical components, we obtained up to $2.7 \mathrm{~W}$ of average power in 900 -fs pulses around $1.45 \mu \mathrm{m}$. In contrast to many other OPOs in this pulse duration regime, the fiber-feedback OPO does not need an active stabilization of the cavity length.

The incorporation of a fiber into a cavity containing bulk components will, in general. introduce substantial losses, mainly at the fiber launch. Nevertheless, a high power conversion efficiency can be achieved if a large parametric gain is available and most of the power of the resonant wave is coupled out directly after the nonlinear crystal. Other intracavity losses then affect only a small portion of the generated power. We achieved a small-signal gain in the order of $90 \mathrm{~dB}$ by applying a high average pump power of up to $8.2 \mathrm{~W}$ from a passively mode-locked Yb:YAG thin disk laser [1] to a periodically poled $\mathrm{LiTaO}_{3}$ (PPLT) crystal, which has a relatively high nonlinearity [2]. The laser is a slightly modified version of the laser described in Ref. [1]. It generates 600 fs-pulses with up to $11 \mathrm{~W}$ at a repetition rate of $35 \mathrm{MHz}$.

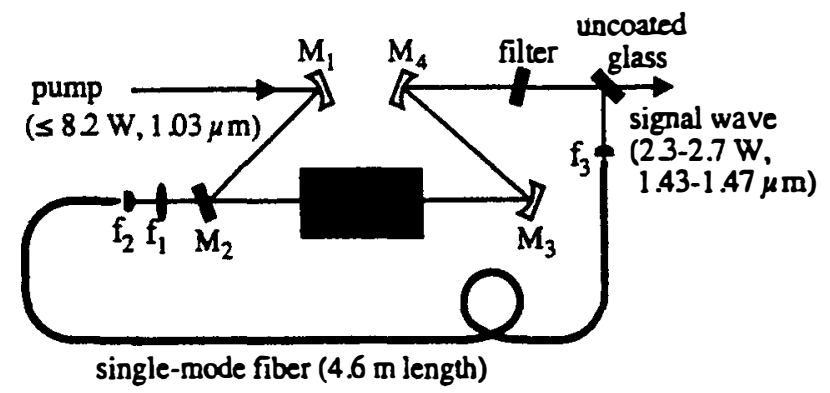

Fig. 1: Setup of the OPO ring cavity. $M_{1}-M_{4}=$ mirrors, $f_{1}-f_{3}$ : lenses, PPLT = crystal of periodically poled $\mathrm{LiTaO}_{3}$. 


\section{Set-up}

The pump beam is focussed with a curved mirror $\left(\mathrm{M}_{1}\right)$ to a waist with $90 \mu \mathrm{m}$ radius in the middle of the PPLT crystal (Fig. 1). The 22-mm long. uncoated crystal is operated at a temperature of $\approx 150^{\circ} \mathrm{C}$ to avoid photorefractive damage. The OPO signal wavelength depends on the period of the poling pattern and the crystal temperature. Our $0.5 \mathrm{~mm}$ thick crystal, fabricated by the same procedure as described for periodic poling of lithium niobate [3], has 8 poled regions of transverse width $1.2 \mathrm{~mm}$, with different grating periods of $28.3 \mu \mathrm{m}-29 \mu \mathrm{m}$, resulting in signal wavelengths between $1429 \mathrm{~nm}$ and $1473 \mathrm{~nm}$ (for $150^{\circ} \mathrm{C}$ crystal temperature). After the nonlinear crystal, the signal wave is collimated and separated from the pump and idler waves by a combination of 3 dichroic elements (mirrors $M_{3}$ and $M_{4}$, filter). One of the two reflected beams from an uncoated glass substrate is used for the signal feedback, while the transmission of $82 \%$ represents the signal output. The feedback light at $\approx 1.45 \mu \mathrm{m}$ is launched into a $4.6 \mathrm{~m}$ long standard telecom fiber which is single-mode at the signal wavelength. The light emerging from the fiber is mode-matched with the lenses $f_{1}$ and $f_{2}$ and fed back into the crystal through the dichroic mirror $M_{2}$, which is highly reflective for the pump wave and transmissive $(70 \%)$ at the signal wavelength.

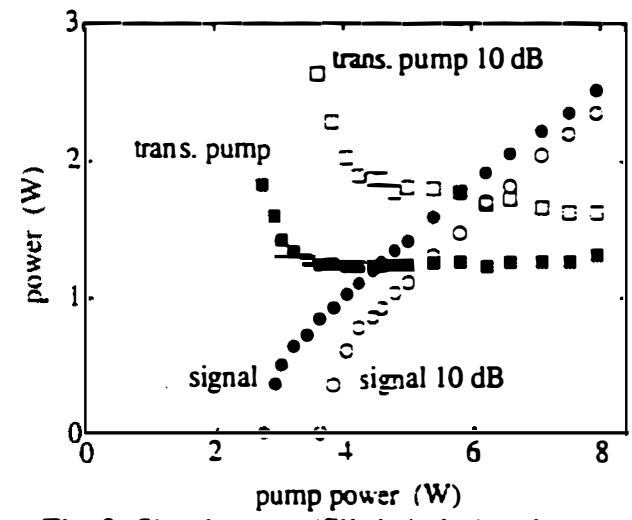

Fig. 2: Signal power (filled sircles) and transmitted pump (filled rectangles) versus pump power for a signal wave of $1429 \mathrm{~nm}$ (grating period $28.3 \mu \mathrm{m}$ ). Open circles and rectangles: same with a 10-dB attenuator in the feedback loop.

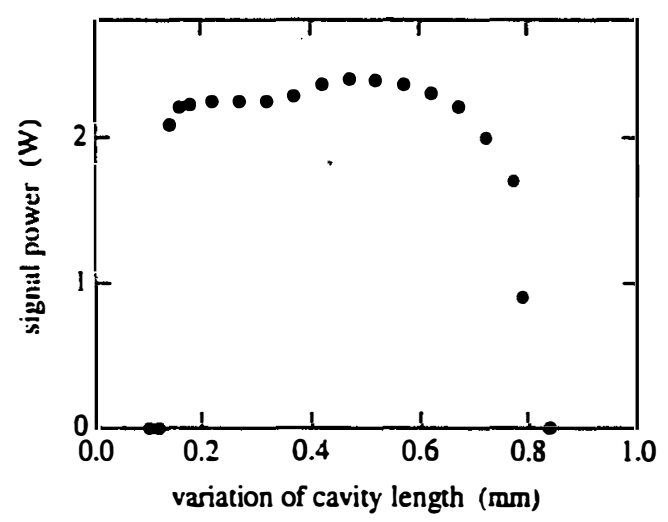

Fig. 3: Variation of signal output power with cavity length.

\section{Results}

The different gratings allow generation of signal output at $1429-1473 \mathrm{~nm}$ with between $2.3 \mathrm{~W}$ and $2.7 \mathrm{~W}$ (measured with a thermal powermeter) for a pump power of $8.2 \mathrm{~W}$ incident on the crystal. Fig. 2 shows the typical performance for one grating. We would expect to obtain even higher signal output powers in the order of $4 \mathrm{~W}$ by reducing the losses of several non-optimized optical components (filter: $5 \%$, glass substrate: one 9-\% reflection suppressed, $15 \%$ at uncoated end faces of the PPLT crystal). The internal pump depletion is up to $=80 \%$ at full power. If required, the idler power (expected to be $1.0-1.1 \mathrm{~W}$ in the present experiment) in the range 3425-3670 $\mathrm{nm}$ could be extracted through an optimized mirror $\mathrm{M}_{3}$.

A notable feature of the fiber-feedback OPO, which results from the high gain and strong output coupling, is the insensitivity of the performance to cavity losses: the maximum output power is reduced by only $6 \%$ if an additional filter with $10 \mathrm{~dB}$ loss at the signal wavelength is inserted at the fiber launch between the glass substrate and lens $\mathrm{f}_{3}$ (Fig. 2). Obviously it is not necessary to minimize the losses in the feedback loop after the output coupler. Lenses $f_{1}-f_{3}$ were uncoated and the polarization of the signal light emerging from the fiber was not controlled.

For a signal wavelength of $1429 \mathrm{~nm}$ and an output power of $1 \mathrm{~W}$, the $\mathrm{M}^{2}$ value was 1.2 in tangential and 1.3 in sagittal direction (the $\mathrm{M}^{2}$ value of the pump beam uas 1.1 ). For $2.5 \mathrm{~W}$ signal pou'er, the $\mathrm{M}^{2}$ value increased to 2.5 in tangential direction and 2.2 in sagittal direction. There might be a significant contribution to this beam quality 
degradation arising from thermally induced bulging of various non-optimized substrates (mirrors $M_{3}, M_{4}$ and the filter) which are heated by idler absorption.

The duration of the signal pulses was measured by intensity autocorrelation. For all poled channels and pump powers, the pulse duration (FWHM) is typically around $700-900$ fs. The spectral width is around 3-4 nm (FWHM). leading to a time-bandwidth product $0.36-0.53$. For $2.5 \mathrm{~W}$ signal power at $1429 \mathrm{~nm}$ (grating period $28.3 \mu \mathrm{m}$ ), we obtain 870 -fs pulses with a spectral width of $2.8 \mathrm{~nm}$, leading to a time-bandwidth product of 0.36 , which is not far from the Fourier limit.

Despite the short pulse duration, the adjustment of the fiber-feedback OPO cavity length is not critical because of the high parametric gain. For example, even if only the leading edge of a signal pulse is temporally overlapped with the pump pulse in the crystal, the high parametric gain still allows for efficient energy extraction. Also note that nonlinear effects in the fiber can lead to a substantial temporal broadening of the seed pulses. Figure 3 shows that varying the round-trip length of the resonator over a range of $0.5 \mathrm{~mm}$ (corresponding to more than one FWHM output pulse width) led to an output power reduction by less than $10 \%$. Within this range, the pulse duration did not change significantly. The central wavelength of the optical spectrum changed less than $0.5 \mathrm{~nm}$, the bandwidth less than $0.3 \mathrm{~nm}$. The operation of the fiber-feedback OPO system is stable over hours, and no signs of crystal damage were observed during all experiments.

Note that the signal pulse energy in the fiber is about two orders of magnitude higher than the soliton energy. As a result of this, we expect the temporal and spectral shape of the pulses after the fiber to be significantly distorted. Nevertheless we obtained near bandwidth-limited output signal pulses because the shor pump pulses create only a narrow time window for gain and the output spectral width is limited by the phase-matching bandwidth.

\section{Summary}

In conclusion. we have demonstrated a novel type of synchronously pumped OPO which is based on feedback through a single-mode fiber. This fiber-feedback OPO concept results in a powerful and efficient, compact and stable source of tunable sub-picosecond pulses. A crucial point is the use of a high parametric gain and strong output coupling, which became possible by the combination of a high pump intensity and a crystal with high nonlinearity. Using non-optimized components, we obtain up to $2.7 \mathrm{~W}$ of signal power in sub-picosecond pulses at a wavelength of 1429-1473 $\mathrm{nm}$. In contrast to other sub-picosecond OPOs, the adjustment of the fiber-feedback OPO cavity length is not critical (range of $0.5 \mathrm{~mm}$ ). The operation is stable over hours. Also the performance is unusually insensitive to additional cavity losses.

In the near future, other signal and idler wavelengths (e.g., signal wavelengths around $1.55 \mu \mathrm{m}$ ) can be generated by modifying the QPM grating period. Also we plan to use fibers with greatly increased mode area [4] in order to exploit soliton formation in the fiber. Signal pulses much shorter than the pump pulses and thus increased output peak powers should be achievable with a fiber-feedback OPO. We also envisage the generation of significantly higher signal and idler average powers because our pump source, a passively mode-locked Yb:YAG thin disk laser, is based on a power-scalable concept.

1. J. Aus der Au, G. J. Spühler. T. Südmeyer, R. Paschotan R. Hövel. M. Moser, S. Erhard. M. Karszewski. A. Giesen. U. Keller, "16.2 W" average power from a diode-pumped femtosecond Yb:YAG thin disk laser." Opt. Lent. 25.859 (2000).

2. J. P. Meyn, M. M. Fejer. "Tunable ultraviolet radiation by second-harmonic generation in periodically poled lithium tantalate," Opt. Lett. 22. 1214 (1997).

3. G. W. Ross, M. Pollnau, P. G. R. Smith, W. A. Clarkson, P. E. Briton. D. C. Hanna, "Generarion of high-power blue light in periodically poled LiNbO," Opt. Lert. 23. 171 (1998).

4. N. G. R. Broderick, H. L. Offerhaus, D. J. Richardson. R. A. Sammut. "Power scaling in passively mode-locked large-mode area fiber lasers." IEEE Photon. Technol. Letr. 10. 1718 (1998). 